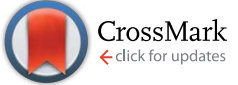

Cite this: Chem. Sci., 2015, 6, 2068

\title{
Brushing up from "anywhere" under sunlight: a universal surface-initiated polymerization from polydopamine-coated surfaces $\uparrow$
}

\author{
Wenbo Sheng, t $^{\mathrm{ab}}$ Bin Li, t $^{\mathrm{ac}}$ Xiaolong Wang, ${ }^{\mathrm{a}}$ Bin Dai, ${ }^{\mathrm{b}}$ Bo Yu, ${ }^{\text {a }}$ Xin Jia*b \\ and Feng Zhou*a
}

\begin{abstract}
We describe a simple yet extremely versatile and generalized surface polymer modification approach based on a surface initiated polymerization from a polydopamine (PDA) layer. PDA deposits on virtually any substrate independent of specific surface chemistries and can act as a photoinitiating layer to initiate the radical polymerization of a variety of (methyl)acrylic/styrene monomers. It does not require any metal/ ligand catalyst, additional photoinitiator or dye sensitizer. Another attractive feature of this novel strategy is the ability to spatially control the architectures (pattern, gradient) of the polymer films by altering the areas of light irradiation. It is also adaptable to large area grafting with an ultra-small amount of monomer solution (a thin monomer solution layer).
\end{abstract}

Received 12th December 2014 Accepted 13th January 2015

DOI: $10.1039 / \mathrm{c} 4 \mathrm{sc03851 \textrm {g }}$

www.rsc.org/chemicalscience peroxides, tertiary amines, etc. ${ }^{\mathbf{1 9 , 2 1 , 2 2}}$ Furthermore, photochemical initiation is usually fast and allows for lithographic patterning of substrates with a myriad of polymers. ${ }^{23,24}$ Recently, metal-based photoredox catalysis triggered via light irradiation has further expanded the surface grafting technologies. ${ }^{25-30}$ However, these reactions usually take place through a radical chain propagation mechanism, and thus require stoichiometric amounts of the transition metal catalyst and initiators. It is highly desirable to develop a more facile approach for generating radical species under mild reaction conditions and avoiding use of transition-metal catalysts and photo initiators which would be expensive or toxic.

Moreover, the development of a simple and generalized strategy for surface modification with multiple classes of materials has proven challenging and, to date, few generalized methods for accomplishing this have been reported. Inspired by the composition of adhesive proteins in mussels, ${ }^{31-33}$ here we report a generalized and metal/ligand-free photopolymerization strategy which is based on a "universal" polydopamine (PDA) adhesive layer for further surface-initiated photopolymerization under simulated sunlight irradiation. Although dopamine has been widely used for surface modifications, its self-polymerization and the subsequent film deposition are kinetically limited. ${ }^{34-36}$ In this work, PDA itself acts as a photoactive species that can generate surface bound radicals to trigger the surface-initiated radical polymerization of a variety of vinyl monomers such as methacrylates, acrylates and styrene onto almost any substrate, representing a new advance in sustainable, environmentally friendly radical reactions, which address the above issues and provide a significantly improved performance in the preparation of thin polymer films. 


\section{Results and discussion}

Scheme 1 outlines the procedure for the preparation of surface attached polymers from PDA-based surfaces. This strategy takes advantage of the PDA deposition on almost any substrate, and the PDA layer can generate surface bound radicals under UV light irradiation that can initiate polymerization. The substrates were immersed in an aqueous solution of dopamine (2 mg mL ${ }^{-1}, 10 \mathrm{mM}$ Tris-HCl, pH 8.5) and the PDA deposition was triggered via ultraviolet irradiation ( $36 \mathrm{~W}$ lamp), leading to the spontaneous deposition of a thin adherent PDA film. This is practically very important for a well-controlled deposition process which is distinguished from the earlier process with slow kinetics, and PDA was activated with further sunlight irradiation to generate radicals for the polymerization of various monomers. Fig. 1a shows the color changes of dopamine solutions under UV and dark conditions at different reaction times. The UV-irradiated solution turned deep dark after 2 hours, while the color change was slow for the nonirradiated solution. Fig. 1b shows the absorption spectra of a dopamine solution under UV irradiation with the increasing absorbance peak at around $410 \mathrm{~nm}$ indicating the growth of PDA with respect to the time. Fig. 1c shows plots of the PDA thickness with respect to the reaction time, analyzed using an ellipsometer. They indicate that the PDA layer thickness was a function of the reaction time and reached up to a value of $c a$. $80 \mathrm{~nm}$ after 10 hours under UV irradiation (red curve). AFM assessment of the PDA films thickness via a scratch test is in good agreement with the data of the ellipsometric thickness (Fig. 1c, blue), and the PDA film (5 h, UV) has a root mean square (RMS) roughness of $2.84 \mathrm{~nm}$ (Fig. S6, ESI $\dagger$ ). While under dark conditions, the polymerization was very slow and the film had a thickness of only about $20 \mathrm{~nm}$ after 10 hours (dark curve). To confirm the mechanism of this photografting process, control polymerizations were conducted on substrates without PDA film or in the absence of light, and both examples demonstrated that the polymerization cannot be triggered without PDA or light, but can be initiated in situ from the PDA surface under simulated "sunlight" irradiation $(300 \mathrm{~W}$ xenon lamp) at $20{ }^{\circ} \mathrm{C}$ (avoiding any thermal reaction during the polymerization). In the absence of PDA, the reaction is retarded. It indicated that the presence of a PDA film is essential for

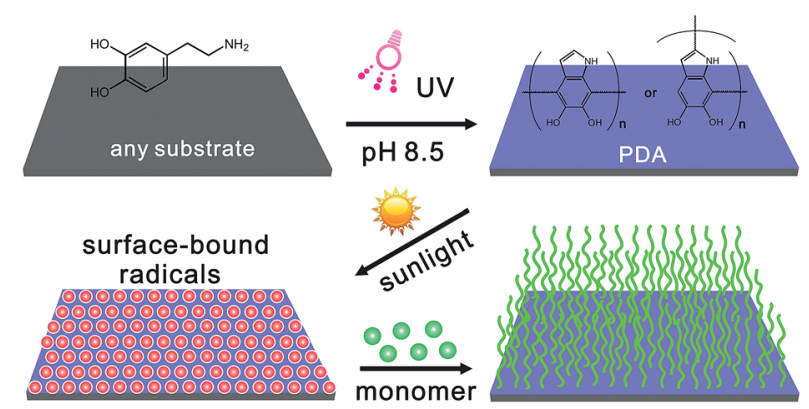

Scheme 1 Schematic illustration of sunlight-induced photografting of polymers from a polydopamine layer that is UV deposited on any substrate.
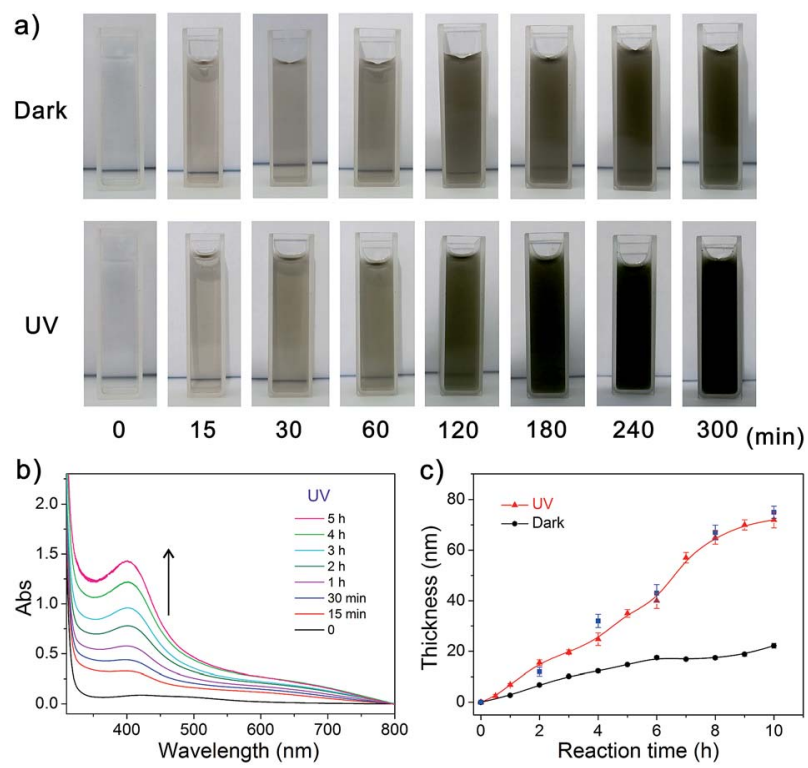

Fig. 1 (a) Color changes of dopamine solutions under dark (upper) and UV irradiation (lower) conditions. (b) Absorption spectral change for a dopamine $\left(2 \mathrm{mg} \mathrm{mL}^{-1}, \mathrm{pH}=8.5\right.$ ) solution upon UV irradiation against the reaction time. (c) Ellipsometric thickness of PDA film with respect to the polymerization time in the dark (dark) and under UV irradiation (red), the blue dots represent the thicknesses obtained via AFM assessments, which agree well with the ellipsometric thicknesses. Lines were added to guide the eyes.

photografting. To confirm the significance of this photochemical process, the kinetics of the same reaction were monitored both with and without light, the polymerization proceeded efficiently under light, but no polymerization was detected under dark conditions. Furthermore, the polymerization did not occur under visible light irradiation when the UV spectrum was filtered out. These experiments indicated that UV light was necessary to bring PDA to its photo-excited state. It is anticipated that this excited PDA* species would generate reactive radicals from PDA through a photosensitized electron transfer process, ${ }^{37-39}$ which could initiate the polymerization of the monomer through a radical chain propagation mechanism that is in analogy with the traditional radical polymerization process. These features provide compelling evidence that this process is a photo-induced radical polymerization. What is the most advantageous aspect of the photo-induced system, and what is different from the traditional surface photo radical polymerization is that the polymerization is initiated in situ on the PDA surface without adding any metal catalyst, photocatalyst or photosensitizer. Furthermore, it would be better to use sunlight instead of UV light because of the possible negative effects of UV irradiation on polymers, in particular biomaterials.

Another distinguished feature of this photopolymerization process is its substrate independence, which allows photografting of polymers on virtually any substrate in the field of materials chemistry. In order to demonstrate its versatility and tolerance to the functional groups and chemical structures of the monomers, we examined the reactivity of different 
(methyl)acrylate and vinyl monomers, such as neutral $\mathrm{N}$-isopropylacrylamide (NIPAm), styrene, cationic 2-(methacryloyloxy)ethyl trimethylammonium chloride (METAC), anionic 3-sulfopropyl methacrylate potassium salt (SPMA) and methacrylic acid sodium salt (MAA-Na). The PDA coated substrates were submerged in the bulk monomer or monomerwater solutions and irradiated with simulated sunlight, and then, the polymers were spontaneously grafted onto various substrates such as inorganic $\mathrm{Al}, \mathrm{Fe}, \mathrm{Ti}$, mica and polymeric polydimethylsiloxane (PDMS), PI and PTFE. Attenuated total reflection infrared (ATR-IR) spectroscopy analysis indicated that the above polymers were successfully grafted onto the surfaces (Fig. S5 in ESI†). Contact angle (CA) measurements also confirmed the formation of PDA and the polymers on the surfaces (Fig. 2a and Table S1 $\dagger$ ), which was demonstrated through the surface polymerization of hydrophilic PSPMA and hydrophobic PS on various substrates coated with thin PDA films. With the PDA layer having medium contact angles, the substrates turned out to be more hydrophilic after the grafting of PSPMA, but more hydrophobic after grafting the PS films. The thickness of the resultant dried polymer films was measured using ellipsometry; the data points are averaged values from separate measurements (Fig. 2b). The AFM image of the PSPMA brushes clearly shows the homogenous growth of PSPMA (with a RMS roughness of $0.25 \mathrm{~nm}$ ) from the PDA coated
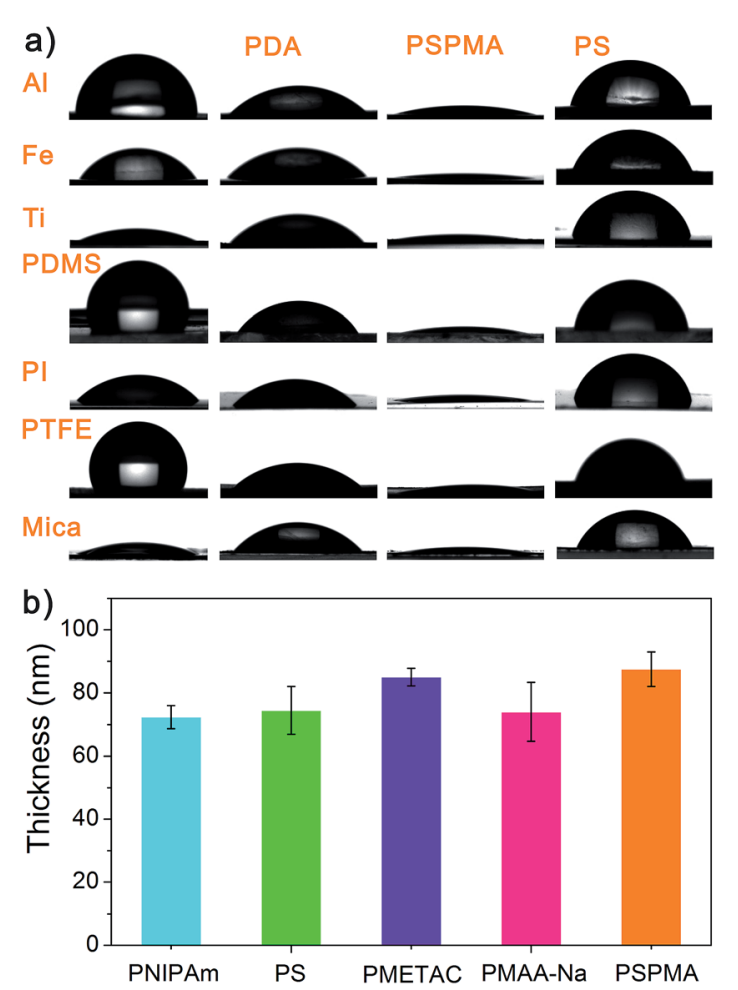

Fig. 2 (a) Pictures of water droplets on several unmodified, polydopamine-coated, PSPMA grafted and PS grafted substrates, including inorganic $\mathrm{Al}, \mathrm{Fe}$, and Ti, the polymers PDMS, PI, and PTFE, and mica. The contact angle values are shown in Table S1. $\uparrow$ (b) Ellipsometric thicknesses of different polymers on a silicon substrate. Reaction conditions: NIPAm (8.8 M, water), 2 h; styrene (bulk), 2 h; METAC ( $80 \%$ in water), 0.5 h; MAA-Na (9.2 M, water), 1.5 h; SPMA (4.0 M, water), 1 h. gold surface (Fig. S6, ESI $\dagger$ ) according to morphology and roughness change. We can prepare functional polymer coatings either in a bulk monomer solution or in aqueous media, enabling the direct polymerization of a broad range of monomers under sunlight without adding any photocatalyst, the way that plants do - using sunlight to construct complex structures. The stability of the PDA/polymer bilayer is important for potential applications, so the sample was immersed in $60{ }^{\circ} \mathrm{C}$ water overnight and washed with various solvents (e.g. water, methanol, acetone, etc.), and no measurable changes in the film thickness were found, indicating the good thermal and solvent stability of these surface grafted polymer films.

The synthesis of patterned polymer films has been demonstrated using lithography (e.g., photo,,$^{23,24,40}$ electron-beam,,$^{41,42}$ scanning probe, ${ }^{43,44}$ etc.), microcontact printing ${ }^{45,46}$ and diffusion controlled strategies. ${ }^{47}$ An attractive feature of this novel method is the possibility to fabricate complex patterns by readily modulating the light to control the reaction areas on the PDA surface. This single-step procedure allows the formation of patterned polymers directly from the pre-patterned and homogeneous PDA surface using a photomask (Fig. $3 a$ and S3†). We can create patterns through two different ways: first, grafting the polymers from a homogeneously covered PDA substrate by masking the sunlight irradiation; or second, grafting them from pre-patterned PDA surfaces that are formed by masking the UV irradiation (Fig. S4†), which is not easily achieved with current methods due to slow kinetics. A variety of features can be obtained, including squares (PMETAC, $54 \mu \mathrm{m}$ in length, Fig. 3b) and circles (PSPMA, ca. $50 \mu \mathrm{m}$ in diameter, Fig. 3c). And most importantly, the non-irradiated areas were kept intact and activated after the first step of polymerization and, therefore, another different polymer can be grafted in a further initiation step. Fig. 3d shows a binary pattern composing two different polymers, i.e., PNIPAm circles (50 $\mu \mathrm{m}$ in diameter) plus backfilled PSPMA. In these cases, polymerization only occurs in the regions where the PDA is exposed to sunlight, resulting in
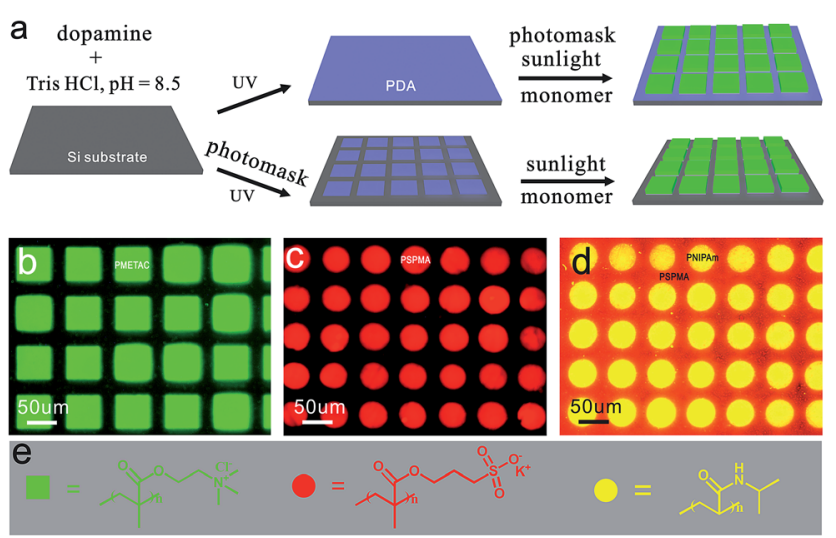

Fig. 3 (a) Outlined procedures for preparing patterned polymers. (b) Fluorescence image of methyl orange-stained PMETAC, (c) Rhodamine 6G-stained PSPMA, (d) binary pattern of acridine-stained PSPMA/ PNIPAm, and the corresponding chemical structures (e). The bright green area is PMETAC, the red area is PSPMA, the yellow area is PNIPAm. 
patterned polymers. Thus, this new photografting method enables the preparation of polymeric films with well-defined and spatially confined chemical functionalities.

The existing surface grafting polymerization methods often have a low space-time yield, which limits their large scale application. In this work, surface attached polymers can be prepared on large surfaces yet using ultra-small amounts of monomer solution as long as the substrates are completely covered with a thin layer of monomer solution and the simulated sunlight spot is large enough. Fig. 4 shows some of the resultant surfaces on large scales. Instead of using a micromachined photomask, we can make photomasks by printing desired patterns on plastic paper with an office use inkjet printer. Deep black ink can stop the sunlight from passing through the covered area but let it through the blank area. So, with an inversely printed Panda image as the photomask, a polymeric "Panda" can be fabricated directly (Fig. 4a). It is also feasible for creating gradient films using a printed black color gradient as a density filter photomask. This allows the direct formation of gradient films in a single step over large areas. Regions of the substrate that were exposed to more light showed an increase in the kinetic rate of polymer formation, resulting in an increase in polymer thickness (Fig. S7†). Fig. 4b shows how this works and plots the height profile of PMETAC along the substrate. The film's thickness shows a linear increase from 70 up to $190 \mathrm{~nm}$ along a $7 \mathrm{~mm}$ distance. Fig. $4 \mathrm{c}$ shows a glass slide that was modified with PS and PSPMA, respectively, each side showing either hydrophobic or hydrophilic properties. Fig. 4d shows the implementation of the grafting strategy on a grape, and one can clearly observe the wetting change after each step.

UV-vis analysis provides a preliminary mechanistic insight into this photomediated process. Presumably, in the PDA deposition, UV light increases the chances for the intramolecular cyclization of oxidised quinone acting as a new monomer for the subsequent PDA buildup, ${ }^{48-50}$ leading to an enhanced reaction rate. For the mechanism of the surface radical polymerization, the interpretation is that in organic chemistry amines are good electron donors which are widely
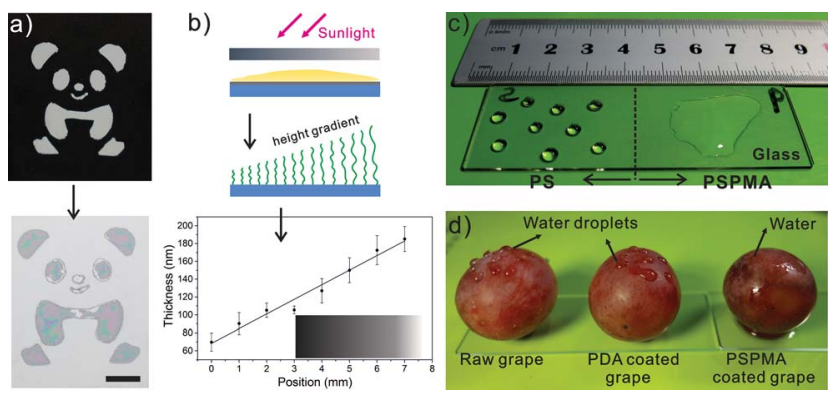

Fig. 4 (a) Optical image of a "Panda" shaped photomask and the PMETAC patterned "Panda" on Si substrate, scale bar: $0.5 \mathrm{~cm}$. (b) Schematic view and thickness gradient of PMETAC on homogeneous PDA coated Si surface (lower) using a printed plastic paper with an optical density gradient (inset), polymerization time $1 \mathrm{~h}$. (c) Photo image of a glass slide with each side modified with either hydrophobic PS or hydrophilic PSPMA. (d) PSPMA grown from a PDA coated raw grape; the grape became completely hydrophilic after photografting of PSPMA. employed for the photochemical single electron transfer (SET) addition of amines to alkenes. ${ }^{37}$ It is known that the PDA surface is covered by a large number of amino functionalities, which provide the possibility of photopolymerization of vinyl monomers. Upon light irradiation (Scheme S1 in ESI $\dagger$ ), surface bound nitrogen-centered radicals are expected to generate photochemically on the PDA surface. Alternatively, such nitrogen-based radicals can undergo proton transfer, producing a protonated amine and a carbon-centered radical that can then couple with the electron-poor monomers, such as (meth)acrylate, which results in the formation of a new chain with a positively charged amine end group. This chain end can either stay protonated or it can become neutral through deprotonation..$^{19,37,51}$ Nevertheless, there is a possibility that the catechol groups could also initiate polymerization under UV light. ${ }^{52}$

On the other hand, the polymerization was completely inhibited in the presence of oxygen, an effective radical quencher, further indicating that the surface-attached polymer chains are formed via a free radical polymerization mechanism. Here, the polymerization mechanism becomes even more complicated due to the lack of detailed information about the PDA structure and the corresponding polymerization mechanism, ${ }^{48,53}$ and the surface polymerization kinetics are highly dependent on parameters such as light, solvent and temperature. Nevertheless, it is clear that this photomediated surface grafting approach is a robust way to prepare well-defined thin polymer films with precisely controlled composition and architectures. Of course, further studies are needed to identify the underlying mechanism.

\section{Experimental}

\section{Polydopamine deposition on silicon surfaces}

The setup used for the deposition of PDA is shown in Fig. S1. $\dagger$ Oxygen plasma (FEMTO) cleaned silicon wafers were immersed in an aqueous solution of dopamine $\left(2 \mathrm{mg} \mathrm{mL}^{-1}\right.$, in $10 \mathrm{mM}$ Tris buffer, $\mathrm{pH}$ 8.5), and the samples were irradiated with a 36 watt fluorescent lamp for a certain time. The same procedure was used for other substrates. Samples were taken periodically for ellipsometry measurements.

\section{General procedure for sunlight-induced photografting of polymers}

Monomer, solvent and the PDA coated surfaces were placed in a sealed vial and degassed by purging with nitrogen for 30 minutes (Fig. S2†). Photopolymerization was initiated upon exposure to a simulated "sunlight". After a set reaction time, the substrates were removed from the vial and washed with water and ethanol, yielding the polymer modified surfaces. See ESI $\dagger$ for details.

\section{Conclusions}

In conclusion, we have developed a new and generalized surface modification approach for fabricating well-defined polymer coatings. Functionalization of the surfaces with self-assembled 
monolayers (SAMs) of initiators is no longer required and direct polymer grafting on surfaces can be realized in a one-step photo-reaction under "sunlight" irradiation, and shows excellent tolerance to many functional groups, which substantially expands the scope of the monomer and solvent choice. In addition, it can be realized on a wide range of substrates that can be planar, curved, and irregular, virtually any chemical substrate, which is more robust than the existing approaches. Polymers can be easily prepared in a rapid, robust manner through the use of a thin and photosensitive polydopamine film. With this facile technique, a wide range of chemical functionalities can be easily coupled to various substrates. These findings are expected to open a new avenue for surface modifications and are very promising for various applications.

\section{Acknowledgements}

This work was financially supported by the NSFC (21434009, 21364010, 21125316) and Key Research Program of CAS (KJZD-EW-M01).

\section{Notes and references}

1 R. Barbey, L. Lavanant, D. Paripovic, N. Schüwer, C. Sugnaux, S. Tugulu and H.-A. Klok, Chem. Rev., 2009, 109, 5437.

2 B. Li, B. Yu, W. T. S. Huck, W. Liu and F. Zhou, J. Am. Chem. Soc., 2013, 135, 1708.

3 M. Baum and W. J. Brittain, Macromolecules, 2002, 35, 610. 4 C. Li and B. C. Benicewicz, Macromolecules, 2005, 38, 5929.

5 I. M. Rutenberg, O. A. Scherman, R. H. Grubbs, W. Jiang, E. Garfunkel and Z. Bao, J. Am. Chem. Soc., 2004, 126, 4062.

6 Q. Ye, X. Wang, S. Li and F. Zhou, Macromolecules, 2010, 43, 5554.

7 M. Husseman, E. E. Malmstro, M. McNamara, M. Mate, D. Mecerreyes, D. G. Benoit, J. L. Hedrick, P. Mansky, E. Huang, T. P. Russell and C. J. Hawker, Macromolecules, 1999, 32, 1424.

8 S. Edmondson, V. L. Osborne and W. T. S. Huck, Chem. Soc. Rev., 2004, 33, 14.

9 D. J. Dyer, Adv. Polym. Sci., 2006, 197, 47.

10 M. Steenackers, S. Q. Lud, M. Niedermeier, P. Bruno, D. M. Gruen, P. Feulner, M. Stutzmann, J. A. Garrido and R. Jordan, J. Am. Chem. Soc., 2007, 129, 15655.

11 M. A. C. Stuart, W. T. S. Huck, J. Genzer, M. Müller, C. Ober, M. Stamm, G. B. Sukhorukov, I. Szleifer, V. V. Tsukruk, M. Urban, F. Winnik, S. Zauscher, I. Luzinov and S. Minko, Nat. Mater., 2010, 9, 101.

12 F. Zhou and W. T. S. Huck, Phys. Chem. Chem. Phys., 2006, 8, 3815.

13 S. Jiang and Z. Cao, Adv. Mater., 2010, 22, 920.

14 H. Ma, J. Hyun, P. Stiller and A. Chilkoti, Adv. Mater., 2004, 16, 338 .

15 W. Senaratne, L. Andruzzi and C. K. Ober, Biomacromolecules, 2005, 6, 2427.

16 B. Li, B. Yu, Q. Ye and F. Zhou, Acc. Chem. Res., 2014, DOI: 10.1021/ar500323p.
17 R. Guo, Y. Yu, Z. Xie, X. Liu, X. Zhou, Y. Gao, Z. Liu, F. Zhou, Y. Yang and Z. Zheng, Adv. Mater., 2013, 25, 3343.

18 D. M. Schultz and T. P. Yoon, Science, 2014, 343, 1239176.

19 K. D. Belfield and J. V. Crivello, Photoinitiated Polymerization, ACS Symposium Series 847, American Chemical Society, Washington, DC, 2003.

20 J. Deng, L. Wang, L. Liu and W. Yang, Prog. Polym. Sci., 2009, 34, 156.

21 D. J. Dyer, J. Feng, R. Schmidt, V. N. Wong, T. Zhao and Y. Yagci, Macromolecules, 2004, 37, 7072.

22 Y. Yagci, S. Jockusch and N. J. Turro, Macromolecules, 2010, 43, 6245.

23 J. E. Poelma, B. P. Fors, G. F. Meyers, J. W. Kramer and C. J. Hawker, Angew. Chem., Int. Ed., 2013, 125, 6982.

24 B. Li, B. Yu and F. Zhou, Macromol. Rapid Commun., 2014, 35, 1287.

25 D. Konkolewicz, K. Schröder, J. Buback, S. Bernhard and K. Matyjaszewski, ACS Macro Lett., 2012, 1, 1219.

26 B. P. Fors and C. J. Hawker, Angew. Chem., Int. Ed., 2012, 51, 8850.

27 A. Anastasaki, V. Nikolaou, Q. Zhang, J. Burns, S. R. Samanta, C. Waldron, A. J. Haddleton, R. McHale, D. Fox, V. Percec, P. Wilson and D. M. Haddleton, J. Am. Chem. Soc., 2014, 136, 1141.

28 J. Xu, K. Jung, A. Atme, S. Shanmugam and C. Boyer, J. Am. Chem. Soc., 2014, 136, 5508.

29 J. Yan, B. Li, F. Zhou and W. Liu, ACS Macro Lett., 2013, 2, 592.

30 S. Dadashi-Silab, M. A. Tasdelen and Y. Yagci, J. Polym. Sci., Part A: Polym. Chem., 2014, 52, 2878.

31 H. Lee, S. M. Dellatore, W. M. Miller and P. B. Messersmith, Science, 2007, 318, 426.

32 J. Sedo, J. Saiz-Poseu, F. Busque and D. Ruiz-Molina, Adv. Mater., 2013, 25, 653.

33 Q. Ye, F. Zhou and W. Liu, Chem. Soc. Rev., 2011, 40, 4244.

34 B. Zhu and S. Edmondson, Polymer, 2011, 52, 2141.

35 M. Kohri, H. Kohma, Y. Shinoda, M. Yamauchi, S. Yagai, T. Kojima, T. Taniguchi and K. Kishikawa, Polym. Chem., 2013, 4, 2696.

36 Z. Ma, X. Jia, J. Hu, G. Zhang, F. Zhou, Z. Liu and H. Wang, Langmuir, 2013, 29, 5631.

37 F. D. Lewis and E. M. Crompton, SET Addition of Amines to Alkene, in CRC Handbook of Organic Photochemistry and Photobiology, ed. W. M. Horspool and F. Lenci, CRC Press, Boca Raton, 2nd edn, 2010.

38 F. D. Lewis and P. E. Correa, J. Am. Chem. Soc., 1984, 106, 194. 39 Z. Su, P. S. Mariano, D. E. Falvey, U. C. Yoon and S. W. Oh, J. Am. Chem. Soc., 1998, 120, 10676.

40 C. Schuh, S. Santer, O. Prucker and J. Rühe, Adv. Mater., 2009, 21, 4706.

41 M. Steenackers, R. Jordan, A. Küller and M. Grunze, Adv. Mater., 2009, 21, 2921.

42 S. J. Ahn, M. Kaholek, W.-K. Lee, B. LaMattina, T. H. LaBean and S. Zauscher, Adv. Mater., 2004, 16, 2141.

43 X. Zhou, X. Wang, Y. Shen, Z. Xie and Z. Zheng, Angew. Chem., Int. Ed., 2011, 50, 6506. 
44 M. E. Welch and C. K. Ober, J. Polym. Sci., Part B: Polym. Phys., 2013, 51, 1457.

45 F. Zhou, Z. Zheng, B. Yu, W. Liu and W. T. S. Huck, J. Am. Chem. Soc., 2006, 128, 16253.

46 B. Li, B. Yu, W. T. S. Huck, F. Zhou and W. Liu, Angew. Chem., Int. Ed., 2012, 51, 5092.

47 J. Yan, B. Li, B. Yu, W. T. S. Huck, W. Liu and F. Zhou, Angew. Chem., Int. Ed., 2013, 52, 9125.

48 N. F. Della Vecchia, R. Avolio, M. Alfè, M. E. Errico, A. Napolitano and M. d'Ischia, Adv. Funct. Mater., 2013, 23, 1331.
49 J. Liebscher, R. Mrowczynski, H. A. Scheidt, C. Filip, N. D. Hadade, R. Turcu, A. Bende and S. Beck, Langmuir, 2013, 29, 10539.

50 W. Xu, X.-M. Zhang and P. S. Mariano, J. Am. Chem. Soc., 1991, 113, 8863.

51 T. G. Ribelli, D. Konkolewicz, S. Bernhard and K. Matyjaszewski, J. Am. Chem. Soc., 2014, 136, 13303.

52 J. Xia, Y. Xu, J. Lin and B. Hu, Prog. Org. Coat., 2008, 61, 7. 53 Y. Liu, K. Ai and L. Lu, Chem. Rev., 2014, 114, 5057. 